\title{
Space-Time Coded OFDM with Low PAPR
}

\author{
Anand Venkataraman, Harish Reddy, and Tolga M. Duman \\ Department of Electrical Engineering, Arizona State University, Tempe, AZ 85287-5706, USA
}

Received 11 January 2005; Revised 25 July 2005; Accepted 1 September 2005

Recommended for Publication by Alex Kot

\begin{abstract}
Recently the use of multiple-input multiple-output (MIMO) orthogonal frequency division multiplexing (OFDM) systems has been proposed for signaling over frequency-selective fading channels. Although various aspects of these systems have been considered in the literature, the problem of the inherent high peak-to-average power ratio (PAPR) is not examined. In this paper, we consider PAPR reduction for MIMO-OFDM systems and propose alternate low-complexity algorithms that can be used in conjunction with the trellis shaping method. We show that a PAPR reduction in the order of $4-5 \mathrm{~dB}$ can be achieved at the cost of a slight reduction in the spectral efficiency. Furthermore, we compare the trellis shaping technique with other PAPR reduction techniques such as tone reservation and partial transmit sequences.
\end{abstract}

Copyright $\odot 2006$ Hindawi Publishing Corporation. All rights reserved.

\section{INTRODUCTION}

Single-carrier modulation together with equalization and multicarrier modulation, such as orthogonal frequency division multiplexing (OFDM), are used to overcome the challenges posed by dispersive channels. OFDM uses a number of subcarriers which are orthogonal to each other. Data is placed on each of the subcarriers and can be recovered at the receiver by exploiting the orthogonality among the subcarriers.

Recently, in addition to the single-input single-output OFDM systems, space-time coded OFDM systems have been receiving significant attention. They were first proposed by Agrawal et al. in order to achieve data rates of $1.5-3 \mathrm{Mbps}$ over a bandwidth of $1 \mathrm{MHz}$ [1], and it is shown that spacetime coding can be used to achieve high data rates at low signal-to-noise ratios (SNRs) over different channels with different multipath delay profiles. In [2], the authors propose a space-time code for a Rayleigh flat fading channel which performs well for various wireless local area network (WLAN) applications. In [3], the authors present an algebraic design framework and propose two approaches for space-time codes in frequency-selective fading channels, one of which employs OFDM. In this scheme, a frequencyselective fading channel is converted into a set of flat block fading channels. Subsequently, an algebraic framework is employed to exploit the diversity available in the block fading channels so as to improve the performance of the system.

Although OFDM has many advantages, it has limitations including high PAPR and carrier frequency offset sensitivity
[4]. Since the complex baseband OFDM signal is formed by the superposition of many sinusoids with different frequencies, the instantaneous power of the resulting signal may be larger than the average power of the OFDM signal exhibiting high peaks. It is important to reduce the PAPR because the high-power amplifiers (HPAs) in a transmitter need to have a linear region that is much greater than the average power, making the HPAs expensive and inefficient. When an HPA with a linear region slightly greater than the average power is used, the saturation caused by the large peaks will induce intermodulation distortion. This distortion increases the bit error rate (BER) and causes spectral widening, which results in adjacent channel interference [5]. Moreover, regulatory bodies specify a peak envelope power limit for a given band, which means that modulation schemes such as OFDM resulting in large peak powers cannot be used directly [6]. For some important contributions in PAPR reduction, see for instance [7-10].

Although many aspects of MIMO-OFDM systems have been addressed, techniques for reducing the PAPR of the resulting OFDM signal are yet to be developed. In our earlier work $[11,12]$, some of the existing single-antenna PAPR reduction algorithms are extended to MIMO-OFDM systems. It is recognized that since the PAPR reduction is achieved without significantly affecting the error rate of the space-time codes and since there are no in-band distortion and out-ofband radiation caused, trellis shaping is a promising technique for PAPR reduction in MIMO-OFDM systems. In this paper, our objective is to propose PAPR reduction techniques 
suitable for MIMO-OFDM systems. We also propose different algorithms of varying complexity levels to be used in conjunction with trellis shaping for MIMO systems as an alternative to the one already being used in the literature, namely, the Viterbi algorithm. Furthermore, to compare the performance of the proposed trellis shaping schemes with other possible alternatives, we also study several other techniques via some examples.

The rest of the paper is organized as follows. In Section 2, trellis shaping for MIMO-OFDM systems is discussed. In Section 3, we present several algorithms that can be used in conjunction with trellis shaping. In Section 4, a comprehensive set of examples are reported to demonstrate that significant PAPR reduction can be obtained with a slight penalty in the spectral efficiency of the MIMO-OFDM system. Finally, conclusions are provided in Section 5.

\section{TRELLIS SHAPING FOR REDUCED PAPR}

A complex baseband OFDM signal can be expressed as

$$
x(t)=\frac{1}{\sqrt{N}} \sum_{l=0}^{N-1} X_{l} e^{j 2 \pi l t / T},
$$

where $x(t)$ is the time domain signal, $X_{l}$ is the complex data symbol on the $l$ th subcarrier, $T$ is the OFDM symbol duration (excluding the guard interval), and $N$ is the number of subcarriers. PAPR is defined as the ratio of the peak power to the average power of the OFDM signal which is given by

$$
\operatorname{PAPR}=\frac{\max |x(t)|^{2}}{E\left[|x(t)|^{2}\right]},
$$

where $E[\cdot]$ is the expected value and $E\left[|x(t)|^{2}\right]$ is the average power of $x(t)$. The statistical distribution of the PAPR is usually characterized by the complementary cumulative distribution function (CCDF) and is given by

$$
\begin{aligned}
\operatorname{CCDF}\left(\operatorname{PAPR}_{0}\right) & =\operatorname{Pr}\left(\operatorname{PAPR}>\operatorname{PAPR}_{0}\right) \\
& =1-F_{\mathrm{PAPR}}\left(\mathrm{PAPR}_{0}\right)
\end{aligned}
$$

where $F_{\mathrm{PAPR}}$ is the cumulative distribution function (CDF) of the PAPR.

Trellis shaping reduces the PAPR of the transmitted sequence by adding a valid convolutionally encoded sequence found using the Viterbi algorithm to it $[13,14]$. In trellis shaping, we use an $(n, k, K)$ convolutional code $C_{s}$, where $n$ is the number of output bits, $k$ is the number of input bits, and $K$ is the constraint length. Other algorithms including list Viterbi and stack algorithm can also be used in conjunction with trellis shaping as will be described later in the paper $[11,12]$. The original data bits can be recovered at the receiver using syndrome former decoding.

PAPR for a MIMO-OFDM signal is defined as the maximum of the PAPRs among all parallel transmit antenna branches. PAPR at the ith transmit antenna is defined as the ratio of the peak power to the average power of an OFDM symbol in that branch. It can be expressed as

$$
\operatorname{PAPR}_{\text {MIMO }}=\max _{1 \leq i \leq n_{t}} \operatorname{PAPR}_{i}
$$

where $\operatorname{PAPR}_{i}=\max \left\{\left|x^{i}(t)\right|^{2}\right\} / E\left[\left|x^{i}(t)\right|^{2}\right]$, and $n_{t}$ is the number of transmit antennas. Here, $E\left[\left|x^{i}(t)\right|^{2}\right]$ denotes the average power of the OFDM symbol from the $i$ th transmit antenna.

\subsection{Trellis shaped space-time coded OFDM with reduced PAPR}

The block diagram of a trellis-shaped space-time coded OFDM system is shown in Figure 1 with $n_{t}$ transmit and $n_{r}$ receive antennas. The main idea of trellis shaping is to add an $(n, k, K)$ convolutionally coded sequence to the information sequence so that the PAPR of the resulting sequence is below an acceptable threshold. Let $G$ be the generator matrix and $H$ the corresponding parity check matrix for the convolutional code used. The convolutionally coded sequence should be removed at the receiver in order to obtain the required information sequence. Also, the convolutional code sequence added at the transmitter has to be selected carefully. To satisfy these two conditions, the following procedure is used $[13,14]$. The input bit sequence, $u$, is grouped into blocks $u_{j}$ of size $(n-k)$ and multiplied by $\left(H^{T}\right)^{-1}$, which is an $(n-k) \times n$ matrix, resulting in blocks $z_{j}$ of length $n$ bits (the need for this operation will be clear after examining the decoding process). Thus, redundancy is introduced at this point and it is given by $1-(n-k) / n$. The output sequence of $z_{j}$ 's is denoted by $\mathbf{Z}$. A valid codeword of the convolutional code has to be selected and added (modulo 2) to Z. To accomplish this, a procedure similar to the decoding of the convolutional codes is followed. The only difference is the specific metric used (which will be described later). Let the path with the least metric correspond to the code sequence $\mathbf{Y}$, which is added (modulo 2 ) to $\mathbf{Z}$ resulting in $\mathbf{Z}^{\prime}$. Through syndrome former decoding, we can remove $\mathbf{Y}$ at the transmitter. The function of the syndrome former decoding can be represented mathematically as

$$
z_{j}^{\prime}\left(H^{T}\right)=z_{j} H^{T} \bigoplus y_{j} H^{T}=u\left(H^{T}\right)^{-1} H^{T}=u
$$

since $y_{j}$ is a valid codeword. The sequence $\mathbf{Z}^{\prime}$ is fed to the space-time encoder and the outputs are transmitted through the $n_{t}$ antennas. At the receiver, the data is space-time decoded, converted to bits represented by $\hat{\mathbf{Z}}$, and then passed to the syndrome former decoder. The output of the decoder is given by $\hat{u}$, which is an estimate of the information sequence $u$.

For each branch in the trellis with label $y_{j}$, we assign a metric $\left|f_{k, d}^{i}\right|$ when proceeding from the current stage, $d-1$, to the next stage, $d$. In MIMO-OFDM, the metric at stage $d$ is the maximum of the metrics amongst the individual transmitting branches and it is given by

$$
\max \left(\left|f_{k, d}^{1}\right|,\left|f_{k, d}^{2}\right|, \ldots,\left|f_{k, d}^{n_{t}}\right|\right) \quad k \in S_{k}
$$

where $\left|f_{k, d}^{i}\right|$ corresponds to the metric at the $i$ th transmit antenna, $1 \leq d \leq N / b_{l}$ is the subblock index, $b_{l}$ is the subblock length given by $n / m$, where $m$ is the number of bits 


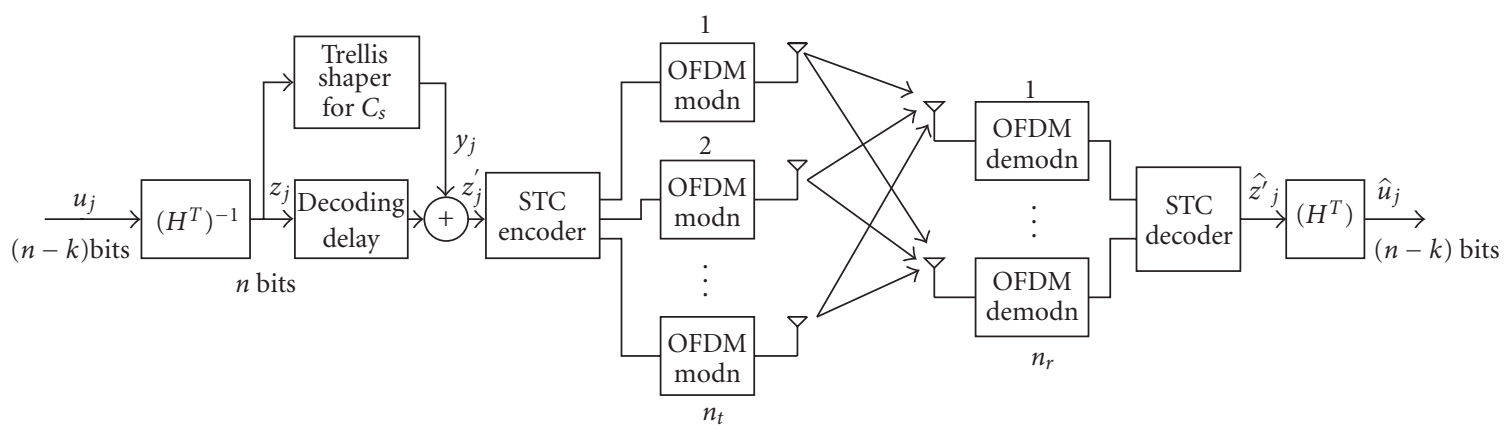

FIGURE 1: Block diagram of trellis shaping for PAPR reduction for multiple antennas using space-time codes.

required to represent 2 complex data symbols, and $S_{k}=$ $\{0,1,2, \ldots, N L-1\}$ with $L$ denoting the oversampling factor. For example, let us consider an $(8,1,2)$ convolutional code. If 16-QAM is used for modulation, we need 4 bits to represent a 16-QAM symbol. Since the number of output bits at each branch is 8 , we can modulate two subcarriers. Therefore, the subblock length is two. Hence, $d$ will have values between 1 and $N / b_{l} . f_{k, d}^{i}$ is computed recursively using [13]

$$
f_{k, d}^{i}=f_{k,(d-1)}^{i}+\sum_{l=(d-1) b_{l}}^{d b_{l}-1} X_{l} e^{j 2 \pi l k / L N},
$$

where the second term on the right-hand side corresponds to a signal obtained using only the subcarriers $(d-1) b_{l}$ to $d b_{l}-1$ modulated by $X_{l}$.

\subsubsection{Computation of the sequence $\mathbf{Y}$}

To find the sequence $\mathbf{Y}$ which will be added modulo-2 to the sequence $\mathbf{Z}$, the Viterbi algorithm (together with the metric given in (7)) may be used [13]. In the case of space-time trellis codes, at the start of each frame (OFDM symbol) the space-time trellis encoder is assumed to be in state 0 . Additionally, to ensure that the trellis ends in the zero state, trellis shaping is not done for all the subcarriers. Instead, it is performed only for $N-N_{f}$ of them, where $N_{f}$ is the number of symbols needed to force the space-time trellis to end in the zero state. The sequence $\mathbf{Z}^{\prime}=\mathbf{Z} \oplus \mathbf{Y}$ along with $N_{f} \times m$ zeros is the input to the space-time encoder for one frame (frame length is selected to be equal to the number of subcarriers, $N)$.

\section{Viterbi and list Viterbi algorithms}

In the Viterbi algorithm, only one surviving path is stored for each state at each time instance. Since we need to minimize $\left|f_{k, d}^{i}\right|$, this process is not optimal. That is, when using the metric without the absolute values as in (7), it is not possible to remove the path(s) with a worse partial metric merging at a certain state while guaranteeing the optimality of the solution. If we could have a similar equation (to (7)) using the absolute values instead, we could say that the use of the Viterbi algorithm would be optimal, however that does not seem to be feasible. Therefore, there might be a possibility that the metric deleted at the stage $d$ can have a better metric at stage $d+1$, when compared to the metric selected at stage $d$ and extended to stage $d+1$. As an alternative to the Viterbi algorithm, to improve the performance of the system, the list Viterbi algorithm with the same metric as in (7) can also be used. By storing more than one path at each state, the list Viterbi algorithm provides alternate paths for searching the best possible sequence resulting in an improved PAPR reduction. However, this adds to the complexity of the algorithm. For example, if two surviving paths are stored at each time instance, then the complexity is twice as much as that of the Viterbi algorithm. Therefore, we propose low complexity approaches such as $M$ - and $T$-algorithms, or tree search algorithms such as the Fano algorithm.

Precisely, if we measure the computational complexity of the algorithm by the number of metrics calculated per OFDM symbol, the Viterbi algorithm calculates $N / b_{l} \times 2^{K-1} \times$ $2^{k}$ metrics and the list Viterbi algorithm with the list size $L_{s}$ is $L_{s}$ times more complex.

\section{Stack algorithm}

Sequential decoding algorithms including the stack algorithm $[15,16]$ can also be employed to find a convolutionally encoded sequence which results in a better PAPR reduction. In the stack algorithm, different paths with different depths are stored based on the value of their corresponding metrics, that is, top of the stack is the path with the least metric. At each stage, the path at the top of the stack is replaced with the $2^{k}$ transitions, where $k$ is the number of inputs to the convolutional encoder at each time instance, and the stack is reordered. Only the paths corresponding to the lowest metrics are retained in the stack. A metric that can be used with the stack algorithm is given by

$$
M_{s}=\max \left(\frac{\left|f_{k, d}^{1}\right|^{2}}{\left\langle\left|f_{d}^{1}\right|^{2}\right\rangle}, \frac{\left|f_{k, d}^{2}\right|^{2}}{\left\langle\left|f_{d}^{2}\right|^{2}\right\rangle}, \ldots, \frac{\left|f_{k, d}^{n_{t}}\right|^{2}}{\left\langle\left|f_{d}^{n_{t}}\right|^{2}\right\rangle}\right),
$$

where $\left\langle\left|f_{d}^{i}\right|^{2}\right\rangle$ denotes the average power at the $i$ th transmit antenna at stage $d, 1 \leq i \leq n_{t}$ is the antenna index, $\left|f_{k, d}^{i}\right|^{2}$ denotes the instantaneous power, $1 \leq d \leq N / b_{l}$ is the subblock index, $b_{l}$ is the subblock length, and $k=\{0,1,2, \ldots, N L-1\}$ is the oversampling index. Since paths with larger depths are consistently replaced with paths of lower depth, the metric 
$M_{s}$ is not computationally efficient, as confirmed by extensive simulations. Therefore, we suggest the alternate metrics, $M_{s_{1}}$ and $M_{s_{2}}$ :

$$
\begin{aligned}
& M_{s_{1}}=\frac{M_{s}}{\sqrt{d}}, \\
& M_{s_{2}}=\frac{M_{s}}{d},
\end{aligned}
$$

where $d$ is the depth of the path.

For these metrics, the cost function is also normalized by the depth of the path (or its square root). Through simulations we have found that, although the alternative metrics are ad hoc, they improve the PAPR reduction performance of the trellis shaping algorithm and reduce the amount of necessary computations for the same PAPR reduction performance. An illustrative example will be provided in the numerical results section.

\section{OTHER LOW-COMPLEXITY ALGORITHMS}

\subsection{M-algorithm}

Since Viterbi algorithm is relatively more complex, we alternatively propose the use of lower-complexity algorithms such as the $M$-algorithm [15] which works similar to the Viterbi algorithm, but it has a smaller number of extended paths at each interval. The metric used is given by (7). The details of the algorithm are as follows.

(i) At depth $d$, consider all $2^{k}$ transitions from each of the $M$ states, where $k$ is the number of input bits to the trellis encoder.

(ii) Select the best $M$ paths with the least metric.

(iii) Go to stage $d+1$ and repeat the process until the depth of the trellis is reached.

The value of $M$ determines the resulting computational complexity which is given by $N / b_{l} \times M \times 2^{k}$. Better PAPR reduction is obtained by selecting a larger value of $M$ as more states are included at each processing interval. When $M$ is equal to the number of states in the trellis encoder, the $M$ algorithm becomes the Viterbi algorithm.

\subsection{T-algorithm}

Another algorithm that can be used to reduce the computational complexity compared to the Viterbi algorithm is the $T$-algorithm [15] which also works similar to the Viterbi algorithm but maintains a variable number of paths based on a threshold, $T$. For the $T$-algorithm, the same metric given in (7) is used, and the number of surviving states at each interval is determined by the closeness of a path with that of the best path. The algorithm is described as follows.

(i) At depth $d$, consider all $2^{k}$ transitions from each of the surviving states.

(ii) Let the path with the best metric be $\alpha$.

(iii) Subtract $\alpha$ from each of the metrics and if the difference is less than a predefined threshold, $T$, accept the transition and go to stage $d+1$. Repeat the process until the depth of the trellis is reached.
The computational complexity of the algorithm depends on $T$ and it can be studied through simulations. In general, the larger the value of $T$, the higher the computational complexity and the better the resulting PAPR reduction.

\subsection{Fano algorithm}

The Fano algorithm [15] can also be used to select the sequence with reduced PAPR through the trellis such that the PAPR of the transmitted sequence is less than a predefined threshold. The algorithm traverses depth first through the trellis and when the metric becomes larger than a predefined threshold at a particular stage, the algorithm backtracks to find an untried path in the preceding stages and proceeds depth first again.

The algorithm calculates metrics of the $2^{k}$ branches at stage $d . M_{s}$ is used as the metric and the path with the smallest metric is found. If this metric is lower than the threshold, we accept the transition and go to stage $d+1$. Otherwise, the algorithm backtracks to stage $d-1$ and finds an untried path with the least metric. If this metric is lower than the threshold, we proceed depth first again through the trellis. If not, we backtrack to stage $d-2$ and repeat the same process. While backtracking, if the root node is reached, that is, we cannot track back any further, we increase the threshold and proceed depth first all over again. Since we do not have the problem of paths of higher depth being replaced by paths of lower depth, that is, the transitions take place only between neighboring stages, we can use $M_{s}$ as the metric. The value of the threshold is determined through simulations in order to optimize the PAPR reduction and minimize the computational complexity. In general, the lower the threshold, the greater the computational complexity; however, the better the PAPR reduction.

\section{Comparison of algorithms used in conjunction with trellis shaping}

The efficiency of the trellis shaping algorithms is calculated in terms of the achieved PAPR reduction and the number of metrics calculated per OFDM symbol. The Viterbi algorithm should perform better than the $M$ - and $T$-algorithms, because at each processing interval transitions from all of the states of the trellis encoder are considered, whereas in $M$ and $T$-algorithms, depending on the value of $M$ and $T$, transitions from only a few states are considered. By increasing the value of $M$ and $T$, the performance of the $M$ - and $T$ algorithms should improve, as we include more states at each processing interval in the trellis.

The stack algorithm is expected to perform better than the Viterbi, $M$-, and $T$-algorithms because the probability of eliminating a good path decreases [11, 12]. Compared to the stack algorithm, the Fano algorithm has a smaller memory requirement. For an appropriate value of the threshold, the Fano algorithm may perform better than the $M$-, $T$ , and Viterbi algorithms because it can find alternate paths through the trellis. When we compare the Fano and stack algorithms that use the same metric, $M_{s}$, and a proper choice 


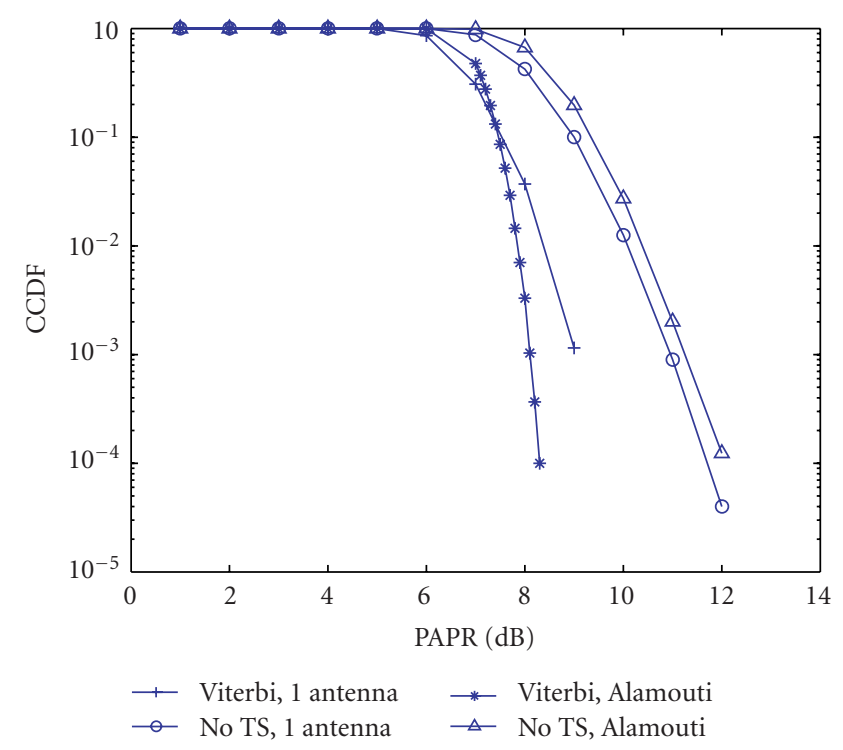

FIgure 2: Comparison of CCDFs of PAPR for the single-antenna case and the two-antenna case with Alamouti scheme. (TS refers to trellis shaping)

of the threshold for a similar PAPR reduction, the Fano algorithm will potentially calculate a smaller number of metrics because the transition takes place only between neighboring nodes. The threshold values for the $T$-algorithm and the Fano algorithm are selected based on a trade-off between computational complexity and PAPR reduction, and they are found based on simulations.

\section{EXAMPLES}

In this section, we present results of the PAPR reduction achieved for space-time coded OFDM system employing the proposed algorithms for use with trellis shaping. The comparison of the PAPR reduction achieved for single- and twoantenna cases for 128 subcarriers using an $(8,1,2)$ convolutional code, and 16-QAM is given in Figure 2. For the case with two transmit antennas, the Alamouti scheme [17] is employed. We observe that the PAPR reduction obtained using the Alamouti scheme is better than the single antenna case.

For the rest of the examples, we consider a space-time coded OFDM system with $N=128$, two transmit antennas, and one receive antenna. To compare the performance of the various algorithms used in conjunction with trellis shaping, we consider an $(8,1,4)$ (8-state), a $(4,1,4)$ (8-state) and an $(8,1,2)$ (2-state) shaping code. In simulations, we use an oversampling factor of 4 which is sufficiently accurate for the discrete samples to model the continuous time signal.

The CCDF of the PAPR for list Viterbi algorithm employing the Alamouti scheme, $(8,1,4)$ convolutional code, and 4-PSK modulation is given in Figure 3. It can be observed that list Viterbi decoding (with list size 4) performs better (by approximately $0.5 \mathrm{~dB}$ ) than the Viterbi algorithm. The CCDF of the resulting PAPR for the Alamouti scheme with a 16-QAM constellation using the $(8,1,4)(8$-state) is shown

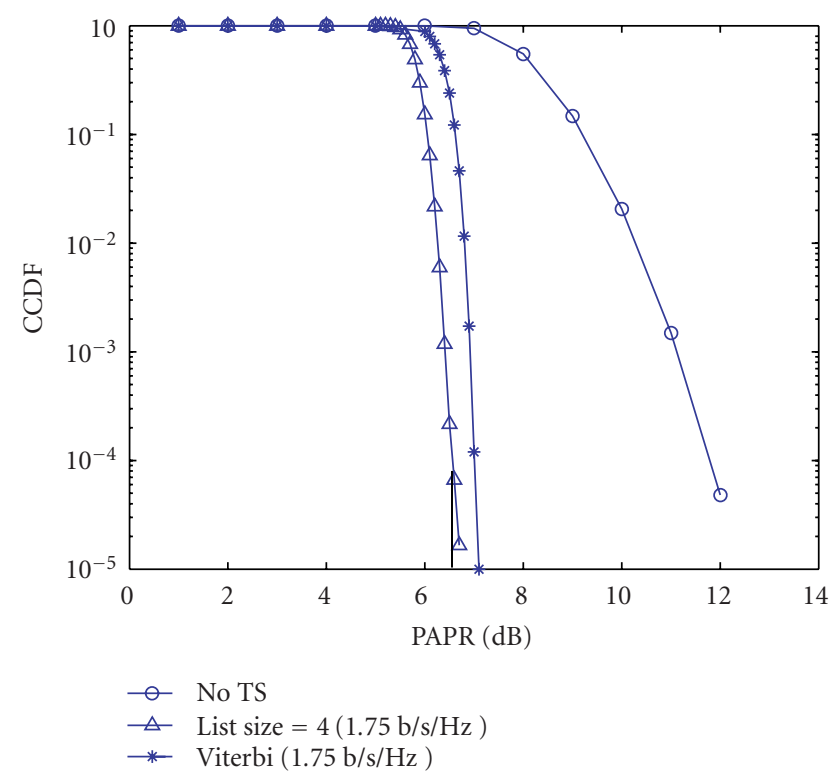

FIGURE 3: Comparison of CCDFs of PAPR for Viterbi decoding and list Viterbi decoding with the Alamouti scheme.

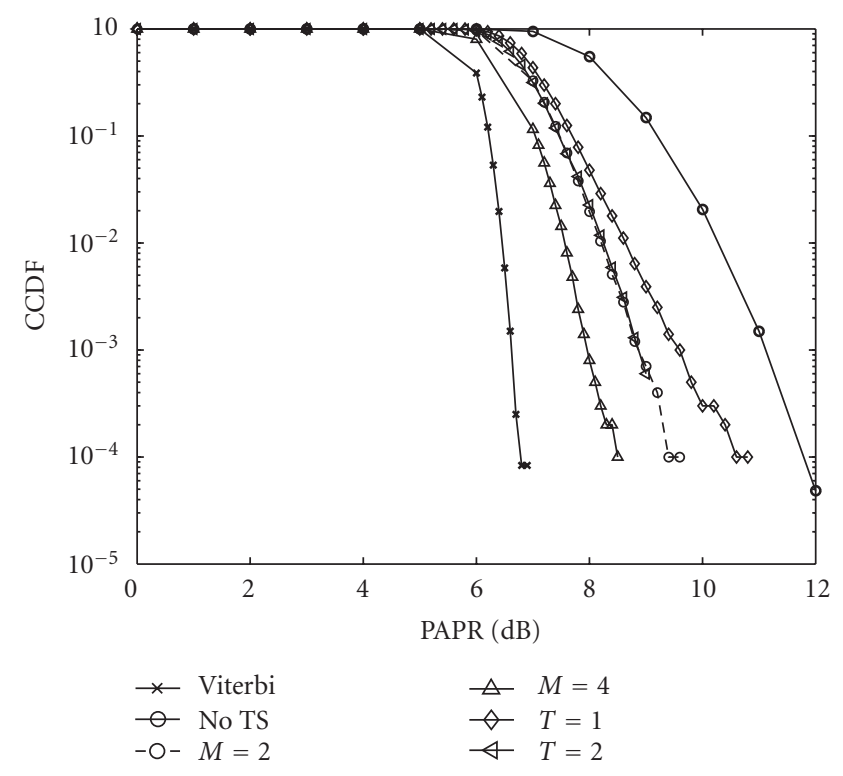

Figure 4: Comparison of the CCDFs of the PAPR between Viterbi, $M$-, and $T$-algorithms for the Alamouti scheme with an $(8,1,4)$ shaping code.

in Figure 4. The original Alamouti scheme has a spectral efficiency of $4 \mathrm{~b} / \mathrm{s} / \mathrm{Hz}$ and the trellis-shaped Alamouti scheme has a spectral efficiency of $3.5 \mathrm{~b} / \mathrm{s} / \mathrm{Hz}$ with a subblock length of two. The Viterbi algorithm achieves a PAPR reduction of about $5 \mathrm{~dB}$ compared to the uncoded system at a CCDF level of $10^{-4}$. At the same CCDF level, compared to the Viterbi algorithm, $M$-algorithm with $M=4$ and $M=2$ is inferior by about $1.7 \mathrm{~dB}$ and $2.5 \mathrm{~dB}$, respectively, in terms of the PAPR 


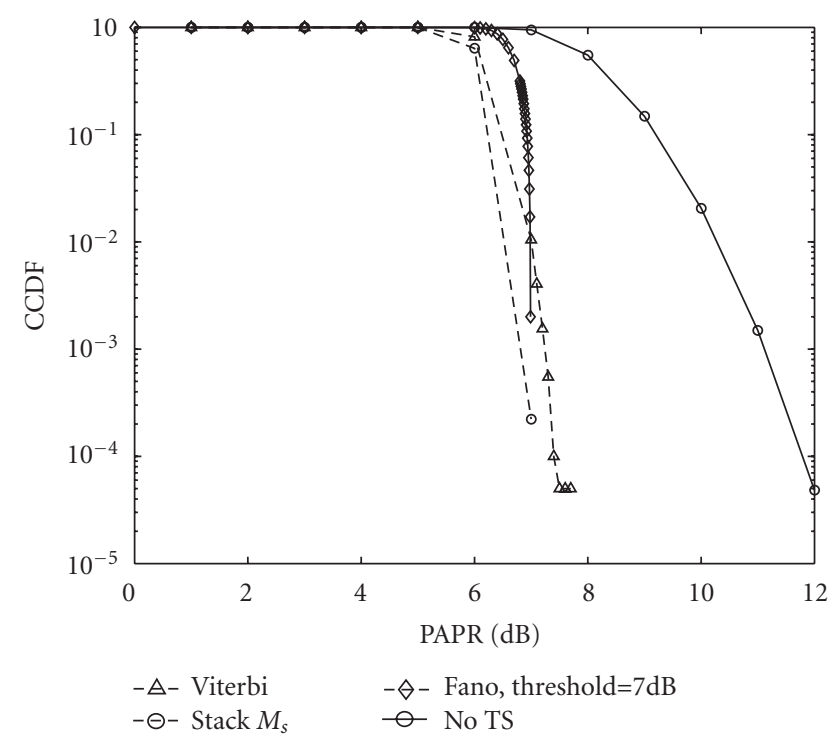

FIgURE 5: Comparison of the CCDFs of the PAPR between Viterbi, Stack, and Fano algorithms for the Alamouti scheme with an $(8,1,2)$ shaping code.

reduction achieved. PAPR reduction achieved by an 8 -state shaping code using $T$-algorithm with $T=1$ and $T=2$ is inferior to the Viterbi algorithm by 3.7 and $2.5 \mathrm{~dB}$, respectively. The computational complexity of the Viterbi algorithm, $M$-algorithm with $M=4$ and $M=2$, and $T$-algorithm with $T=1$ and $T=2$ is $1024,512,256,172$, and 268 , respectively. It can be seen that as the computational complexity is reduced there is degradation in the PAPR reduction.

The CCDF of the resulting PAPR for the Alamouti scheme with a 16-QAM constellation using the $(8,1,2)(2$ state) is shown in Figure 5. For a 2-state shaping code using the stack algorithm with the metric $M_{s}$, we obtain a PAPR reduction of about $5 \mathrm{~dB}$ at a CCDF level of $10^{-4}$. With the Fano algorithm, we obtain a reduction in PAPR similar to that of the stack algorithm using metric $M_{s}$ at a CCDF of $10^{-4}$. At the same CCDF level, Viterbi algorithm achieves a PAPR reduction of about $4.5 \mathrm{~dB}$. The computational complexities for the Viterbi, stack, and Fano algorithms with the specific parameters selected are 256, 550, and 225 per OFDM symbol, respectively.

The CCDF of the PAPR using the alternate metrics for stack algorithm using an $(8,1,2)$ convolutional code is given in Figure 6. We see that using these alternate metrics the loss in PAPR reduction is within $1 \mathrm{~dB}$. On the other hand, the computational complexity using $M_{s}, M_{s_{1}}$, and $M_{s_{2}}$ are 550, 210, and 155 per OFDM symbol, respectively. Therefore, using $M_{s_{1}}$ reduces the computational complexity by half when compared to $M_{s}$. However, the reduction in PAPR is degraded by only around $0.5 \mathrm{~dB}$ at CCDF level of $10^{-3}$. Stack algorithm with $M_{s_{1}}$ performs similar to the Viterbi algorithm and the compuational complexities are comparable. By using $M_{s_{2}}$, we achieve further reduction in computational complexity but with trade-off in PAPR reduction as also apparent in the figure.

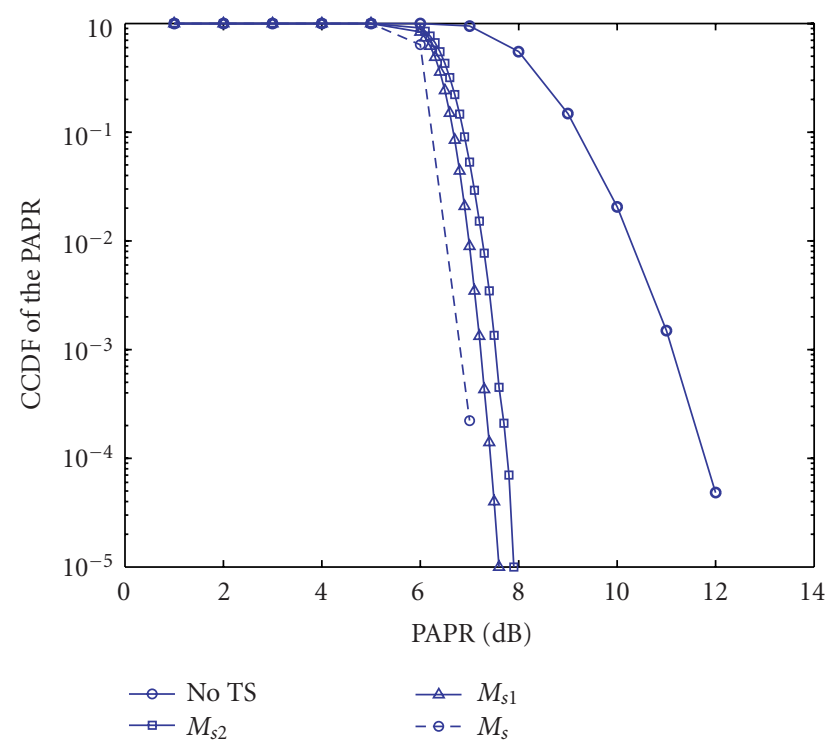

FIGURE 6: Comparison of the CCDFs of the PAPR for alternate metrics used with stack algorithm.

The computational complexity and the PAPR at a CCDF level of $10^{-3}$ for the different algorithms with $(8,1,2)$ and $(8,1,4)$ shaping codes are summarized in Table 1 . From the simulations it is noted that, when the Fano algorithm is employed for a 2-state shaping code, the number of metrics computed per OFDM symbol is 225 for a threshold of $7 \mathrm{~dB}$ (averaged over a large number of simulations) and 170 for a threshold of $7.5 \mathrm{~dB}$. Clearly, using alternate metrics for stack algorithm reduces the computational complexity. Thus, a trade-off exists between the selected threshold and the computational complexity.

We now consider the $(4,1,4)(8$-state) shaping code. The CCDFs of the resulting PAPRs for the Alamouti scheme with a 4-PSK constellation using $M$-, $T$-, and Fano algorithms are shown in Figure 7. The spectral efficiency of the uncoded system is $2 \mathrm{~b} / \mathrm{s} / \mathrm{Hz}$. The spectral efficiency of the trellis-shaped space-time coded OFDM system is $1.5 \mathrm{~b} / \mathrm{s} / \mathrm{Hz}$. The subblock length is two. We see from the plots that the reduction in PAPR using $M$-algorithm with four states is very close to that of the Viterbi algorithm. This may be because of the increase in the redundancy of the convolutional code. At a CCDF level of $10^{-3}$, the PAPR is $6.4 \mathrm{~dB}$ for the Viterbi algorithm. At the same CCDF level, the PAPRs for the $M$ algorithm with $M=4, T$-algorithm with $T=1$, and Fano algorithm with a threshold of $7 \mathrm{~dB}$ are $6.6,8$, and $7 \mathrm{~dB}$ respectively. The computational complexities for the Viterbi algorithm, $M$-algorithm with $M=4, T$-algorithm with $T=1$, and Fano algorithm with a threshold of $7 \mathrm{~dB}$ are 1024, 512, 232 , and 270 per OFDM signal, respectively.

In order to illustrate the performance obtained with space-time trellis codes, the CCDF of the resulting PAPRs for the 4 -state space-time trellis code from $[18]$ with a $(4,1,4)$ (8-state) shaping code using $M$-, $T$-, and Fano algorithms are shown in Figure 8. The subblock length is two. QPSK 
TABLE 1: Comparison of the computational complexity and the PAPR at a CCDF of $10^{-3}$ for the different algorithms used in conjunction with trellis shaping with subblock length $\left(b_{l}\right)=2$ and $N=128$ for the Alamouti scheme.

\begin{tabular}{lcc}
\hline Trellis shaping algorithms & Number of metrics computed & PAPR at CCDF $=10^{-3}(\mathrm{~dB})$ \\
\hline Viterbi $(8,1,2)$ & 256 & 7.4 \\
Stack $M_{s}(8,1,2)$ & 550 & 6.8 \\
Stack $M_{s 1}(8,1,2)$ & 210 & 7.4 \\
Stack $M_{s 2}(8,1,2)$ & 155 & 7.8 \\
Fano Threshold $=7 \mathrm{~dB}(8,1,2)$ & 225 & 6.9 \\
Viterbi $(8,1,4)$ & 1024 & 6.6 \\
$M=2(8,1,4)$ & 256 & 9.0 \\
$M=4(8,1,4)$ & 512 & 8.0 \\
$T=1(8,1,4)$ & 172 & 9.7 \\
\hline
\end{tabular}

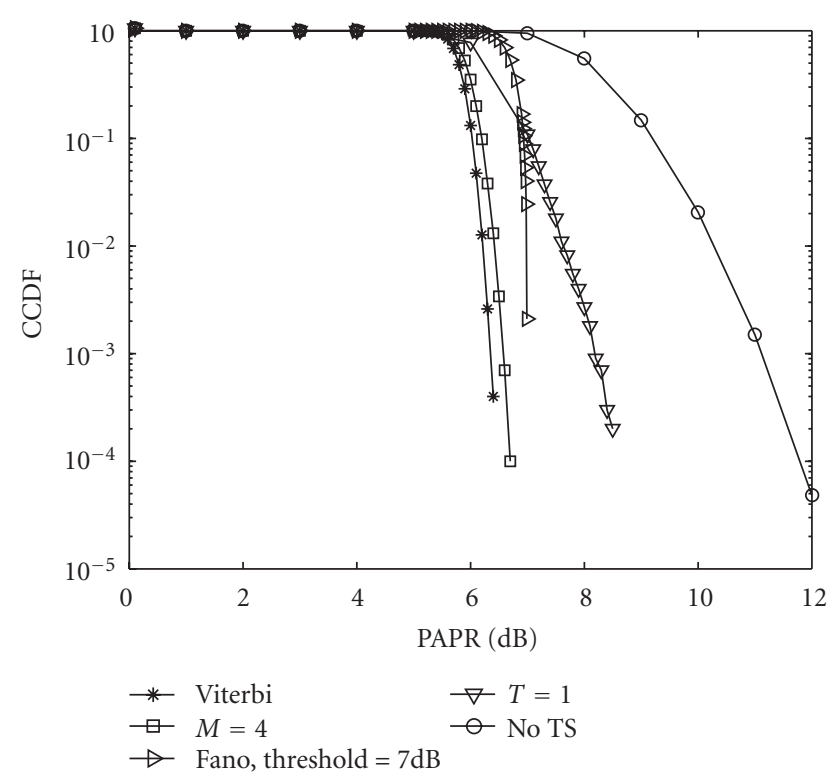

Figure 7: Comparison of the CCDFs of the PAPR for the Alamouti scheme using a $(4,1,4)$ shaping code.

constellation has spectral efficiency of $2 \mathrm{~b} / \mathrm{s} / \mathrm{Hz}$ and hence, the trellis-shaped space-time coded OFDM system has a spectral efficiency of $1.5 \mathrm{~b} / \mathrm{s} / \mathrm{Hz}$. We observe that the reduction in the PAPR using the $M$-algorithm with four states is very close to the one achieved by the Viterbi algorithm. At a CCDF level of $10^{-3}$, the PAPRs for the Viterbi algorithm, $M$ algorithm with $M=4, T$-algorithm with $T=1$, and Fano algorithm with a threshold of $7 \mathrm{~dB}$ are $7.2,7.4,8$, and $7 \mathrm{~dB}$, and the resulting computational complexities are 1024, 512, 244, and 296 per OFDM symbol, respectively. Hence, low complexity algorithms can be used instead of Viterbi and stack algorithms for reduction in computational complexity without degrading the performance significantly.

In order to illustrate the effectiveness of the trellis shaping for MIMO OFDM systems, we also study the use of several other techniques, namely, the use of partial transmit sequences [20] and tone reservation [21]. The comparison of the CCDF of the PAPRs obtained using trellis

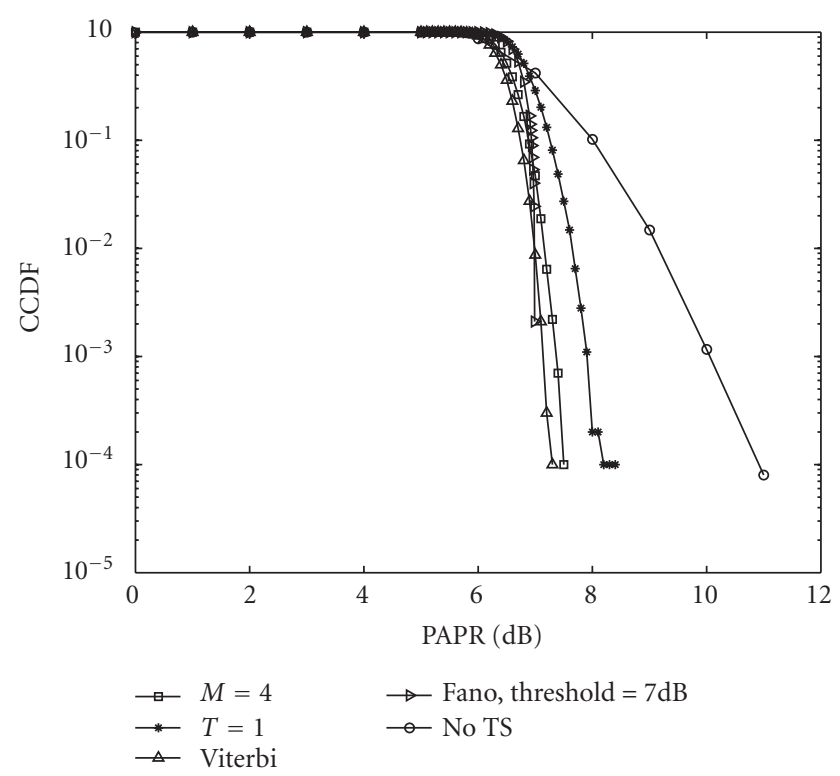

FIGURE 8: Comparison of the CCDFs of the PAPR for the space-time Trellis code from [18] using a $(4,1,4)$ shaping code.

shaping, tone reservation, and partial transmit sequences for $N=128$ using the $(8,1,4)$ code and space-time codes from $[17,19]$ are shown in Figures 9 and 10, respectively. Here, we use a 4-PSK constellation which results in a spectral efficiency of $2 \mathrm{~b} / \mathrm{s} / \mathrm{Hz}$. All three PAPR reduction techniques result in a spectral efficiency of $1.75 \mathrm{~b} / \mathrm{s} / \mathrm{Hz}$. As can be seen from the figures, trellis shaping performs comparable or better than partial transmit sequences and tone reservation in terms of PAPR reduction, while tone reservation performs better than the partial transmit sequences.

The bit error rate of the three PAPR reduction techniques under a quasi-static flat Rayleigh fading channel, which is constant during the transmission of an OFDM symbol and changes independently from one symbol to another, is given in Figure 11. In trellis shaping, the degradation in the BER is due to the error in the syndrome former decoding and is the same irrespective of the algorithm used. In partial transmit sequences, if one of the rotational factors is decoded 


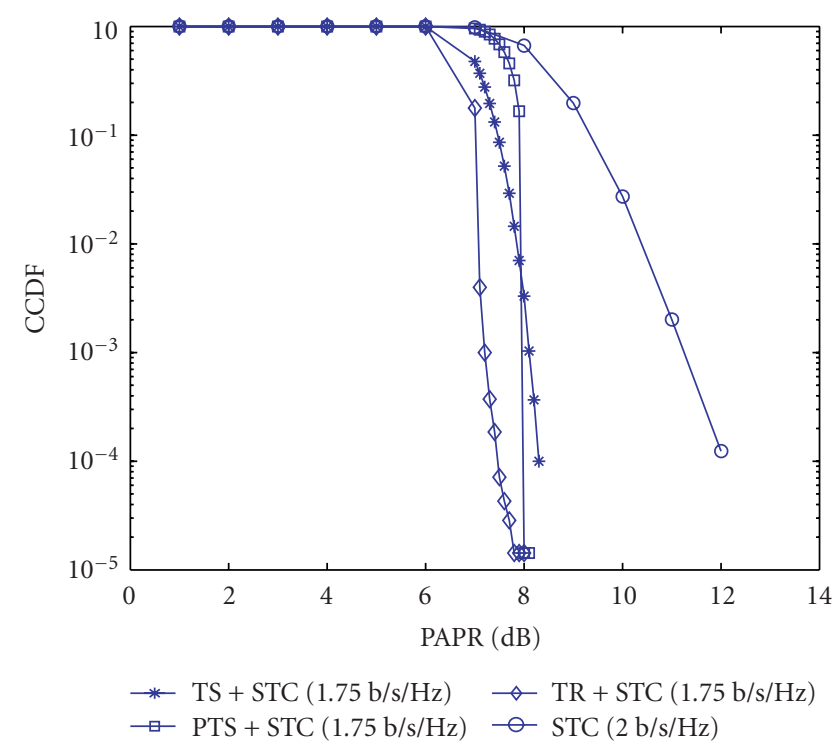

FIgUre 9: Comparison of the CCDFs of the PAPR for the STC between trellis shaping, partial transmit sequence, and tone reservation for STTC from [19] with $N=128$. (TR:tone reservation, PTS: partial transmit sequence).

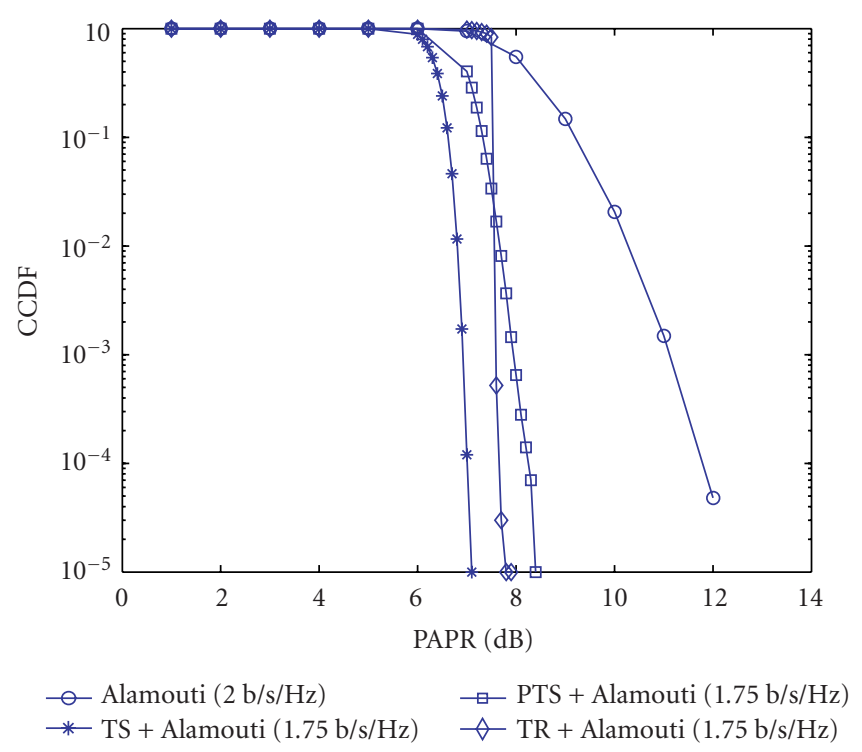

FIgUre 10: Comparison of the CCDFs of the PAPR between trellis shaping, partial transmit sequence and tone reservation for the Alamouti scheme with $N=128$.

incorrectly, then the entire subblock is decoded erroneously, and this results in the BER degradation. There is no BER degradation using tone reservation, as no side information is transmitted to decode the data at the receiver.

In partial transmit sequences, each iteration involves the rotation of the $V$ subblocks ( $N$ subcarriers are divided into $V$ subblocks) and the computation of an IFFT of size $N L$ where $L$ is the oversampling factor. Hence, the complexity for each iteration is given by $N$ rotations, $N L \log N L$ additions, and $N L \log N L$ multiplications per IFFT. In tone reservation, the

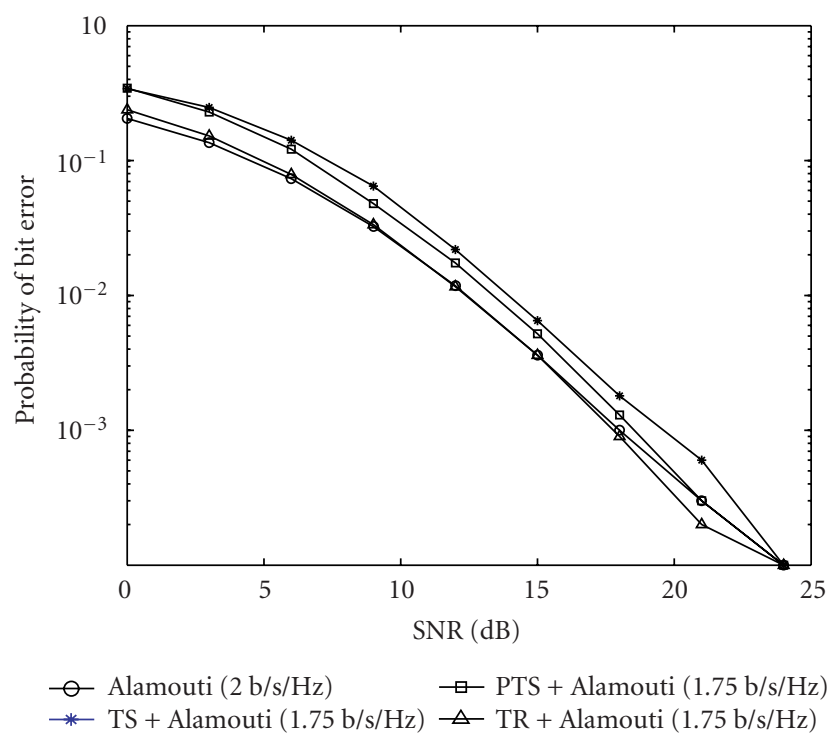

FIGURE 11: Comparison of the BER for three PAPR reduction techniques with $N=128$.

complexity for each iteration is given by the $N L$ comparisons to locate the peak, $N L$ multiplications to scale the signal $\delta(t)$, and $N L$ additions/subtractions for reducing the peak at the given location.

\section{CONCLUSIONS}

In this paper, we have considered the problem of PAPR reduction for MIMO-OFDM systems. We have extended the use of trellis shaping to MIMO-OFDM systems using spacetime trellis and space-time block codes. In addition to the commonly used Viterbi algorithm in the trellis shaping, we have proposed the use of several other algorithms that provide lower complexity solutions and/or improved PAPR reduction performance. We have observed that with a slight reduction in the spectral efficiency of the system, it is possible to achieve a PAPR reduction in the order of $4-5 \mathrm{~dB}$. We have also compared the performance of trellis shaping against tone reservation and partial transmit sequences which are alternative low-complexity approaches. Our proposed lowcomplexity algorithms provide a computational complexity and PAPR reduction performance trade-off.

\section{ACKNOWLEDGMENTS}

This work was supported in part by NSF CAREER Award CCR-9984237 and by a grant from the Connection One Center. Also, part of this work was performed while the third author was on a sabbatical leave at Bilkent University, Turkey.

\section{REFERENCES}

[1] D. Agrawal, V. Tarokh, A. Naguib, and N. Seshadri, "Spacetime coded OFDM for high data-rate wireless communication 
over wideband channels," in Proc. 48th IEEE Vehicular Technology Conference (VTC '98), vol. 3, pp. 2232-2236, Ottawa, Ontario, Canada, May 1998.

[2] S. Rouquette-Leveil and K. Gosse, "Space-time coding options for OFDM-based WLANs," in Proc. 55th IEEE Vehicular Technology Conference (VTC '02), vol. 2, pp. 904-908, Birmingham, Ala, USA, May 2002.

[3] H. El Gamal, A. R. Hammons Jr., Y. Liu, M. P. Fitz, and O. Y. Takeshita, "On the design of space-time and space-frequency codes for MIMO frequency-selective fading channels," IEEE Transactions on Information Theory, vol. 49, no. 9, pp. 22772292, 2003.

[4] T. Pollet, M. V. Bladel, and M. Moeneclaey, "BER sensitivity of OFDM systems to carrier frequency offset and Wiener phase noise," IEEE Transactions on Communications, vol. 43, no. 234, pp. 191-193, 1995.

[5] R. O’Neill and L. B. Lopes, "Envelope variations and spectral splatter in clipped multicarrier signals," in Proceedings of 6th IEEE International Symposium on Personal, Indoor and Mobile Radio Communications (PIMRC '95), vol. 1, pp. 71-75, Toronto, Ontario, Canada, September 1995.

[6] V. Tarokh and H. Jafarkhani, "On the computation and reduction of the peak-to-average power ratio in multicarrier communications," IEEE Transactions on Communications, vol. 48, no. 1, pp. 37-44, 2000.

[7] H. Ochiai and H. Imai, "Performance analysis of deliberately clipped OFDM signals," IEEE Transactions on Communications, vol. 50, no. 1, pp. 89-101, 2002.

[8] X. Huang, J. Lu, J. Zheng, K. B. Letaief, and J. Gu, "Companding transform for reduction in peak-to-average power ratio of OFDM signals," IEEE Transactions on Wireless Communications, vol. 3, no. 6, pp. 2030-2039, 2004.

[9] R. van Nee and A. de Wild, "Reducing the peak-to-average ratio of OFDM," in Proceedings of 48th IEEE Vehicular Technology Conference (VTC '98), vol. 3, pp. 2072-2076, Ottawa, Ontario, Canada, May 1998.

[10] X. Wang, T. T. Tjhung, and C. S. Ng, "Reduction of peakto-average power ratio of OFDM system using a companding technique," IEEE Transactions on Broadcasting, vol. 45, no. 3, pp. 303-307, 1999.

[11] H. Reddy, "Peak to average power reduction for coded MIMOOFDM," Master's thesis, Arizona State University, Tempe, Ariz, USA, December 2003.

[12] H. Reddy and T. M. Duman, "Space-time coded OFDM with low PAPR," in Proceedings of IEEE Global Telecommunications Conference (GLOBECOM '03), vol. 2, pp. 799-803, San Francisco, Calif, USA, December 2003.

[13] W. Henkel and B. Wagner, "Another application for trellis shaping: PAR reduction for DMT (OFDM)," IEEE Transactions on Communications, vol. 48, no. 9, pp. 1471-1476, 2000.

[14] H. Ochiai, "A novel trellis-shaping design with both peak and average power reduction for OFDM systems," IEEE Transactions on Communications, vol. 52, no. 11, pp. 1916-1926, 2004.

[15] C. Schlegel, Trellis Coding, IEEE Press, Piscataway, NJ, USA, 1997.

[16] A. J. Viterbi and J. K. Omura, Principles of Digital Communication and Coding, McGraw-Hill, New York, NY, USA, 1979.

[17] S. M. Alamouti, "A simple transmit diversity technique for wireless communications," IEEE Journal on Selected Areas in Communications, vol. 16, no. 8, pp. 1451-1458, 1998.

[18] Q. Yang and R. S. Blum, "Optimum space time convolutional codes," in Proceedings of IEEE Wireless Communications and Networking Conference (WCNC '00), vol. 3, pp. 1351-1355, Chicago, Ill, USA, September 2000.
[19] V. Tarokh, N. Seshadri, and A. R. Calderbank, "Space-time codes for high data rate wireless communication: performance criterion and code construction," IEEE Transactions on Information Theory, vol. 44, no. 2, pp. 744-765, 1998.

[20] S. Müller, R. Bäuml, R. Fischer, and J. Huber, "OFDM with reduced peak-to-average power ratio by multiple signal representation," Annals of Telecommunications, vol. 52, no. 1-2, pp. 58-67, 1997.

[21] J. Tellado, Multicarrier Modulation with Low PAR: Applications to DSL and Wireless, vol. 587 of Kluwer International Series in Engineering and Computer Science, Kluwer Academic, Boston, Mass, USA, 2000.

Anand Venkataraman received the B.E. (honors) degree in electrical engineering from Birla Institute of Technology and Science, Pilani, India, in 2002, and the M.S. degree in electrical engineering from Arizona State University in 2004. He is currently with Qualcomm Inc., San Diego. His research interests include multicarrier communications and spread-spectrum communications.

Harish Reddy received his B.E. degree from BMS College of Engineering, Bangalore, India, in 2000, and the M.S. in electrical engineering from Arizona State University in 2003. He is working in the Wireless Research Group of Tata Consultancy Services since May 2004. His research interests include OFDM, signal processing for wireless communications, and MIMO systems.
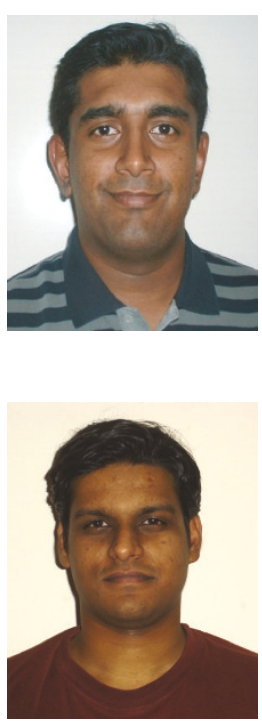

Tolga M. Duman received the B.S. degree from Bilkent University in 1993, and the M.S. and Ph.D. degrees from Northeastern University, Boston, in 1995 and 1998, respectively, all in electrical engineering. Since August 1998, he has been with the Electrical Engineering Department of Arizona State University first as an Assistant Professor (1998-2004), and currently as an Associate Professor. His current research inter-

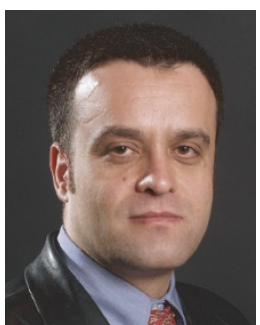
ests are in digital communications, wireless and mobile communications, channel coding, turbo codes, coding for recording channels, and coding for wireless communications. He is the recipient of the National Science Foundation CAREER Award, IEEE Third Millennium Medal, and IEEE Benelux Joint Chapter Best Paper Award (1999). He is a Senior Member of IEEE, and an Editor for IEEE Transactions on Wireless Communications. 